\title{
Unresolved abdominal mass
}

\author{
Shrestha B, Baidya JL \\ Section of Urology, B\&B Kathmandu University Hospital, Gwarko
}

\begin{abstract}
A case of huge enhancing solid mass in the abdomen observed in 30 years old gentlemen is reported. Patient was worked up and managed conservatively outside valley with the provisional diagnosis of an appendicular lump.

When examined, he was found to have huge intra abdominal mass. Interestingly when the genitalia was examined he was found to have underdeveloped empty scrotum without testes in it which he was yet not bothered. This simple examination gave us a straight clue to come up with the diagnosis of malignancy in undescended testes.

Laparotomy was performed and the mass was excised. After 6 weeks postoperatively he was subjected for the chemotherapy. After3 cycles of chemotherapy CT scan showed complete disappearance of retroperitoneal lymphadenopathy and serum tumor markers have come down to normal level.
\end{abstract}

Key words: Germ cell tumor (GCT), Seminoma, Non seminomatous germ cell tumor, (NSGCT), Cryptorchidism, Alpha fetoprotein (AFP), Beta human chorionic gonadotrophin (BHCG), Lactate dehydrogenase (LDH)

\begin{abstract}
testicular tumor may become metastatic and $A$ manifest with large retroperitoneal and/or chest lesions, while the primary tumor is non palpable or may not be bothersome to the patient. A case of huge solid abdominal mass in 30 years old male was ultimately diagnosed to have metastatic testicular cancer after meticulous examination and history taking before that he had been treated in the line of appendicular lump. We have reported this case because many a times it is not uncommon to miss the diagnosis not just because of unavailability of diagnostic tools but also lack of thorough clinical work up and taking for granted the scenario.
\end{abstract}

Seventy five percent of the seminomas are localized (stage I) when they are first diagnosed however, 15\% tend to have regional lymph nodes and 5-10\% already had juxta nodes or visceral metastases ${ }^{1}$.

Testicular malignancy is notorious for delayed presentation because of the fact that the subject is least bothered about self-examination and fails to alert physician of any mass. At last but not the least owing to inability to diagnose the entity in time and the case being managed as the conditions like epididymo-orchitis or testicular trauma.

Having malignancy on one side, chances of acquiring malignancy in normal testes on the other side is about $5-10 \%$. However, the risk rises to $25 \%$ when the subject has bilateral cryptorchidism ${ }^{2}$.
Various tumor markers are available in the form of alpha fetoprotein (AFP), beta human chorionic gonadotrophin (bHCG), Lactate dehydrogenase (LDH), Placenta like alkaline phosphotase (PLAP) which not only help in making the diagnosis but also helps in formulating management plan. Their sensitivity and specificity varies according to the type of testicular tumour. Yolk sac elements secrete AFP; an elevated level rules out pure seminoma, despite possible contrary histopathologic findings at orchidectomy. Lactate dehydrogenase (LDH) is a less-specific marker for GCTs, but levels can correlate with overall tumor burden.

\section{Case report}

Thirty years old gentleman presented to us with the history of pain abdomen and abdominal mass of one and half month duration. Mass was gradually increasing in size and he had normal bladder and bowel habits. Patient was investigated with ultrasound of the abdomen which was suggestive of appendicular lump. He was then decided to manage conservatively with broad spectrum antibiotics and analgesics. Despite these, his symptoms didn't get better hence he was investigated further with CT scan of the abdomen and was referred to us for the diagnostic work up and further management.

\footnotetext{
Correspondence

Dr. Binod Shrestha

Registrar, Urology,

B \& B Kathmandu University Teaching Hospital

P. O. Box: 10829, Kathmandu, Nepal

E-mail: drbinshrestha@yahoo.com
} 
When we examined him we found a huge lump measuring $15 \times 15 \mathrm{cms}$ globular in nature, non tender at the centre of abdomen. It was slightly mobile and there was no audible bruit over it. There was no supracalvicular adenopathy. Interestingly when we examined his groin and genitalia, scrotum was poorly developed and there were no palpable testes. When we examined the groin there wasn't any palpable lump either. While enquiring he acknowledged that he had never felt his testes in his life.

With these findings we investigated him further with serum tumor markers which revealed bHCG to be $560 \mathrm{mIU} / \mathrm{ml}(<5 \mathrm{mIU} / \mathrm{ml})$. LDH and alpha fetoprotein were found to be with in normal limits. CT abdomen revealed lobulated heterogeneous mass in the retroperitoneum measuring $13 \times 12 \mathrm{cms}$, displacing inferior venecava (IVC) slightly towards right side and extending from L1 to S3. Chest X-ray which was done was normal.

The final diagnosis of Right germ cell tumor of the undescended abdominal testes was made. With this we planned for the laparotomy. At laparotomy $15 \times 15 \mathrm{cms}$ mass at hypogastrium was found to be adherent with the small bowel and omentum. It was excised after cross clamping at it's pedicle with 2-0 silk.

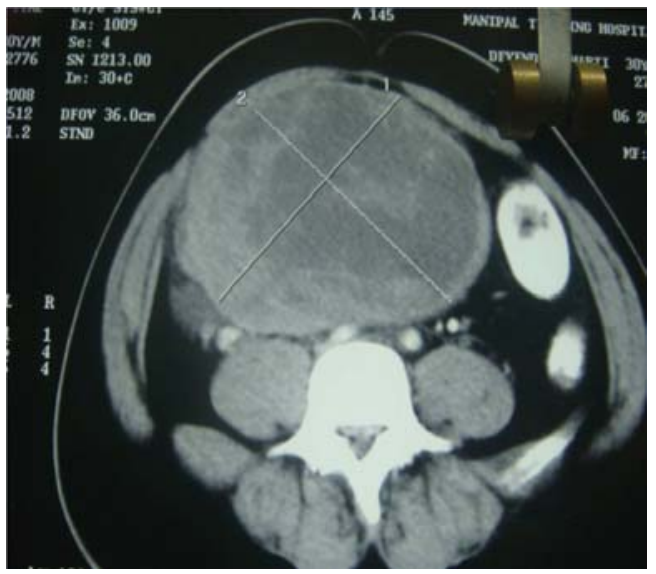

Fig 1: Axial CT scan showing heterogenous enhancement of the tumour in undescended testes
Liver and omentum and rest of the bowel were normal. There was no ascites, contra lateral testis was found to be very soft in consistency measuring about $2 \times 2 \mathrm{cms}$ lying at the left iliac fossa. It was brought down through the deep inguinal ring after dividing the posterior peritoneum and adherent fibres. It could not be brought down beyond the external ring where it was anchored and orchidopexy was performed. Besides, there was $8 \times 8 \mathrm{cms}$ hard retroperitoneal adenopathy around the renal hilum which was untouched.

Patient had uneventful post operative recovery and was discharged on the sixth post operative day. On follow up the histopathology was found to be a classic seminoma and the hormone parameters were; AFP- $1.8(<8.5 \mathrm{ng} /$ $\mathrm{ml})$, HCG- $1.5(<5 \mathrm{mIU} / \mathrm{ml})$, LDH- 40 after second post operative weeks.

He was later planned for six cycles of chemotherapy. After three cycles of chemotherapy (Bleomycin $30 \mathrm{U}$ (0.25-0.5 U/kg) IV, Etoposide $100 \mathrm{mg} / \mathrm{m} 2 \mathrm{IV}$, Cisplatin $20 \mathrm{mg} / \mathrm{m} 2 \mathrm{IV}$ ) hormone profile was repeated again which was found as follows: AFP-1.6ng/ml $(<8.5)$ and HCG- $0.1 \mathrm{mIU} / \mathrm{ml}(<5.0)$.

CT abdomen which was done after three cycles of chemotherapy revealed merely a pin head size of residual retroperitoneal lymphadenopathy. With this the patient has been well counseled for frequent follow up.

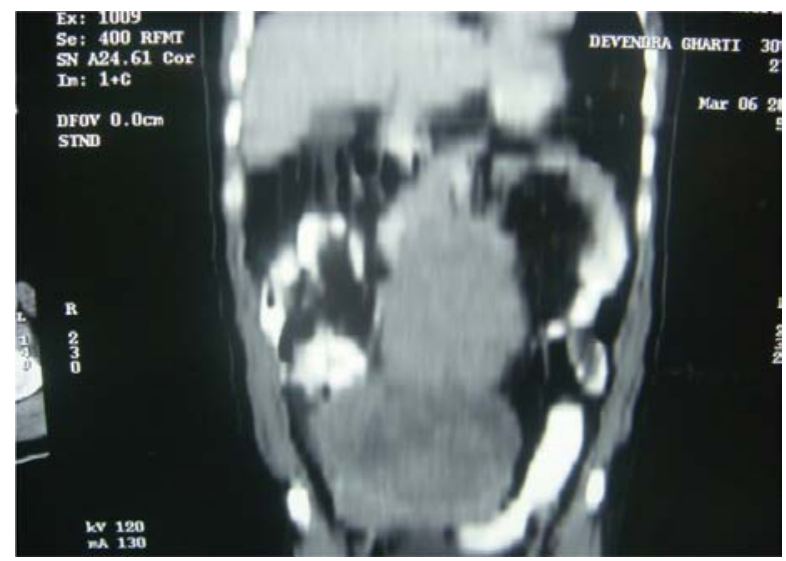

Fig 2: Coronal section of CT scans showing retroperitoneal mass above the primary mass 


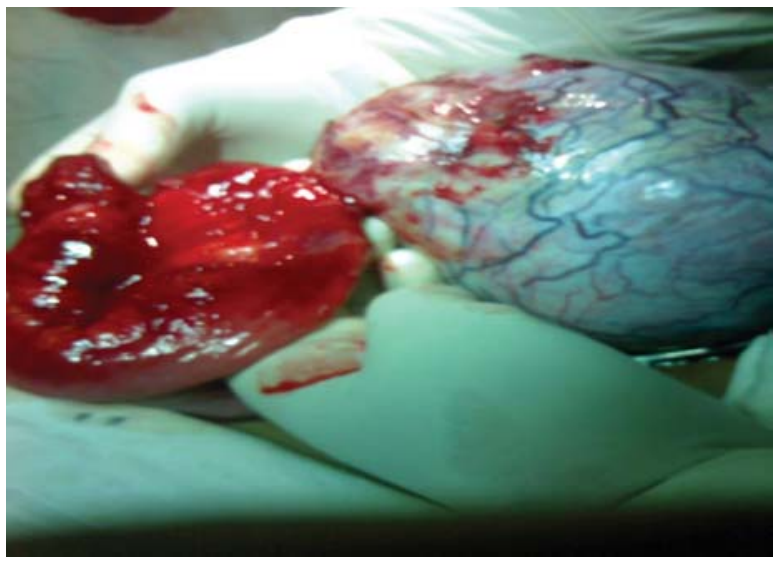

Fig 3: Testicular mass bluntly dissected off from the densely adherent small bowel

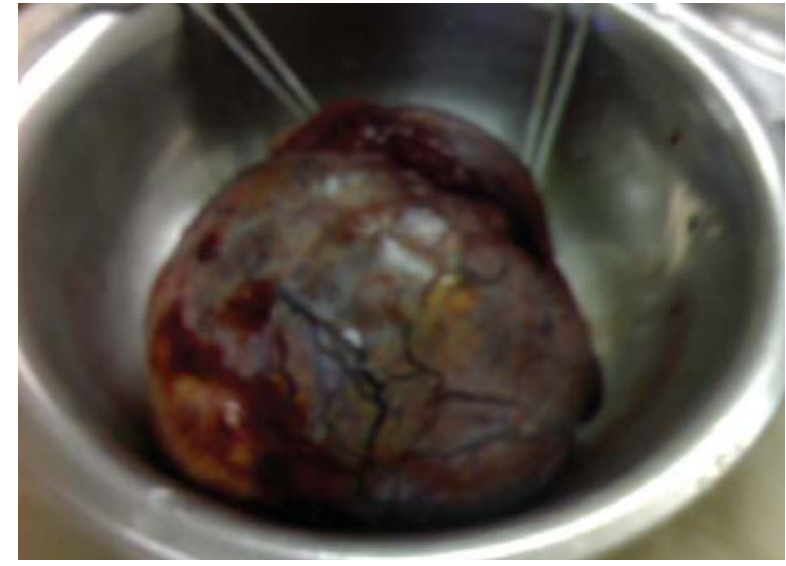

Fig 4: Testicular mass after excision

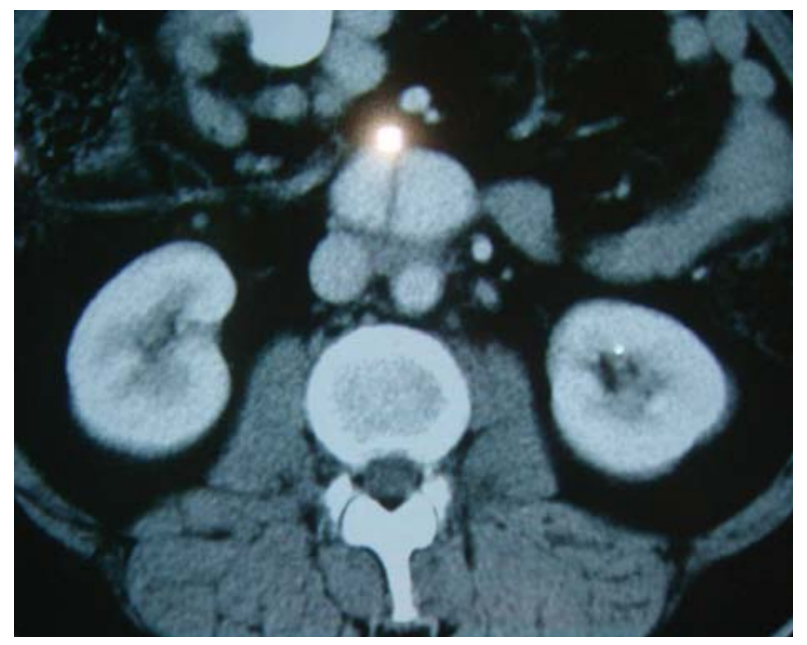

Fig 5: Axial CT scan showing tiny residual retroperitoneal mass in-between IVC and Aorta

\section{Discussion}

Testicular germ cell tumours (GCT) are rare, occurring in only $1-2 \%$ of all male malignancies and occurring in 1 of 250 men by age 65 years. However, GCT is the most common malignancy in men aged 15-35 years ${ }^{1}$.

Germ cell tumor is 10 - to 40 -times more common in cryptorchids however, $10 \%$ Germ cell tumor have a history of cryptorchidism. Undescended testes with intra abdominal testes are more likely to be seminomas. However, after orchidopexy for the intra abdominal testes; it is more likely to be Non seminomatous germ cell type. It has been stated that the chances of development of malignancy in the contralateral normally developed testes in which there is history of malignancy in the undescended testes is $5-10 \%$. However, if there is history of bilateral cryptorchidism with the history of malignancy of testes on one side then chances of having malignancy in the contralateral testes will be as high as $25 \%$. It has been proved that early orchidopexy though help in earlier detection, it doesn't alter the risk of malignancy ${ }^{1}$.

In 1996 Daugaard and associates advocated that biopsy be obtained from the contralateral testis at the time of orchidectomy because of possible carcinoma in situ on the contralateral testes in 5\% of GCT patients ${ }^{2}$. However in 1997 Herr and Sheinfeld were not in favour of biopsy because $95 \%$ of the times the contralateral testes would be normal and possible 5\% involvement of contralateral testis can be effectively treated at a later time ${ }^{3}$.

Various imaging modalities are available to diagnose and stage the condition. Classically Scrotal ultrasonography shows hypoechoic intratesticular mass in seminoma and at times with some calcification and cystic changes though mainly in non seminomas. 
CT scanning of the abdomen and the pelvis is a very sensitive tool for the metastatic evaluation which definitely helps in planning the management. CT scan of the chest can usually be omitted unless there is an abnormal CXR finding.

The ideal treatment for the bulky stage 2 tumour and stage 3 tumour should be radical orchidectomy followed by adjuvant chemotherapy. At present, four cycles of chemotherapy is said to be sufficient which include Cisplatin and Etopside though earlier on six cycle of chemotherapy used to be instituted. However, three cycles of chemotherapy has equally good results when Cisplatin, Etoposide and Bleomycin regimen is used ${ }^{4}$. In poor risk patients and refractory to the conventional chemotherapy regimen, Ifosfamide and Vinblastine can be tried.

Regarding retroperitoneal lymphadenopathy $90 \%$ of the residual mass tend to be very small and simply fibrosed. However, if it is more than $3 \mathrm{cms}$ then the chances of residual seminoma is as high as $40 \%$ and in those circumstances surgical excision is warranted ${ }^{5}$.

For the bulky tumour, doing a radical surgery and chemotherapy is not sufficient. Patient has to be followed up very frequently. During each visit apart from history and clinical examination including that of the contra lateral testes, serum markers and radiological imaging has to be performed (Ideally with CT scanning). Patients has to be followed up every two months for the first year, every three months for the second year, every four months for the third year, every six months for the fourth year and annually thereafter. Metastasis in the liver or the central nervous system, initially or at recurrence, is currently the only proven adverse prognostic factor ${ }^{6}$.

\section{Conclusion}

Germ cell tumour though sounds alarming has a very good prognosis with a high cure rate provided it is picked up earlier. Every case of testicular tumour has to be dealt with a multidisciplinary approach where apart from surgeons, pathologist, chemo and radio oncologist and radiologists come up with definite management plan. Metastatic work up has to be thorough once there is suspicion of testicular tumor and pre orchidectomy hormone profile has to be sent for future reference and monitoring. After the primary management, the need of regular follow up should be properly informed to the patient. If facility is available then of course the issue regarding fertility and possible sperm banking has to be discussed with the patient because adjuvant chemotherapy and Radiotherapy can adversely affect spermatogenesis. At last but not the least, Genitalia examination which is often ignored should always be made a part of clinical examination.

\section{References}

1. Richie JP. Neoplasms of the Testis. In: Walsh PC, Retik AB, Vaughan ED Jr, Wein AJ, eds. Campbell's Urology. 7th ed. Philadelphia, Pa: WB Saunders; 1998. P. 2411-52.

2. Daugaard G, Giwercman A, Skakkebaek NE. Should the other testis be biopsied? Semin Urol Oncol 1996 Feb;14(1):8-12.

3. HerrHW,SheinfeldJ.Isbiopsyofthecontralateral testis necessary in patients with germ cell tumors? J Urol 1997 Oct;158(4):1331-4.

4. Joseph C, Presti JR. Genital Tumours. In: Tanagho EA, McAninch JW, editors. Smith's General Urology. 17 $7^{\text {th }}$ edition. New York: Lange Medical Books/McGraw-Hill, Medical Publishing Division; 2008. P. 375-82.

5. Motzer R, Bosl G, Heelan R. Residual mass: an indication for further therapy in patients with advanced seminoma following systemic chemotherapy. J Clin Oncol 1987 Jul;5(7):106470 .

6. Gholam D et al. Advanced seminoma - treatment results and prognostic factors for survival after first-line, cisplatin-based chemotherapy and for patients with recurrent disease: a singleinstitution experience in 145 patients. Cancer 2003 Aug 15;98(4):745-52. 